\title{
Development of Micro Fiber Edge Detection System Based on Grey System Theory
}

\author{
Yan Wan \\ School of Computer Science and Technology, \\ Donghua University, \\ 200051 Shanghai, China
}

\author{
Shuo-Lei Sun \\ School of Computer Science and Technology, \\ Donghua University, \\ 200051 Shanghai, China
}

\author{
Zi-Lin Shi \\ School of Computer Science and Technology, \\ Donghua University, \\ 200051 Shanghai, China \\ zilinshi@163.com
}

\begin{abstract}
Based on the grey system theory, Nib lack algorithm and hole filling algorithm, the automatic detection system of micro fiber edge is developed. The mathematical model and design idea of the algorithm are introduced, and the design results are presented. The experimental results show that the design can extract complete and accurate micro fiber edge information.
\end{abstract}

Keywords-fiber, edge detection, grey system theory, grey prediction model

\section{INTRODUCTION}

With the development of economy and technology, fiber has important application in fields of society. The composition and quality of the fiber determines its performance and price, and the integrity and continuity of the fiber edge has a direct impact on the accuracy of subsequent recognition and the accuracy of the calculation of blending ratio [1]. But in the process of fiber collection, there are uneven illumination, background noise, focusing and other factors. Micro fiber images inevitably appear some problems that there is no obvious distinction between the target and the background, adhesion, fracture contour and so on [2]. Edge detection algorithm based on the grey system theory that is combining the grey prediction model with Niblack algorithm to get the edge information of the fiber and for the phenomenon that there are some false edge inside of fibers using the hole filling algorithm to extract the complete fiber edge can avoid the phenomenon which contains fiber edge discontinuity, false edges and fibronectin which cannot be determined the contour. Starting from the algorithm principle, this paper which is based on the grey system theory of fiber edge detection system design describes the system design ideas and implementation details. The experimental results show the effectiveness of the design.

\section{Mathematical Model of Grey System Theory}

The grey system theory which was founded by Deng Julong, a famous scholar in China in 1982, is a new method to study the uncertainty of the poor information. Professor Deng Julong according to the definition of system divided the system into three categories, namely the white system, black system and grey system. Among them, the white system refers to the system that information is completely known, the black system refers to the system that information is completely unknown [3]. The grey system refers to the system that part of the information is known and part of the information is unknown. Commonly using mathematical statistical modeling all needs lots of data as modeling data, and according to the priori law or statistical law deal with the problem [4]. And the grey system theory which only needs a small amount of sample without knowing the transcendental character of the original data can establish mathematical model, and can find the factors that influence the development trend of system, so as to complete the prediction of the system.

Grey prediction model GM $(1,1)$ is an important part of grey system theory, which based on the idea of data fitting to establish differential equation model, namely on the basis of the data sequence of nonnegative after generation transform rendering index law of exponential, seeks generating function in the exponential function [5]. The main algorithm modeling process is shown below.

Suppose the original data sequence is

$$
\begin{gathered}
\mathrm{x}^{(0)}=\left(\mathrm{x}^{(0)}(1), \mathrm{x}^{(0)}(2), \ldots, \mathrm{x}^{(0)}(\mathrm{n})\right) \\
\left(\mathrm{x}^{(0)}(\mathrm{k}) \geq 0, \mathrm{k}=1,2, \ldots, \mathrm{n}\right) .
\end{gathered}
$$

The original data sequence is first-order accumulated to weaken the randomness of the original data sequence and generate a new sequence of data [6]. Let $\mathrm{x}^{(1)}$ is the sequence which is first-order accumulated by $\mathrm{x}^{(0)}$ and the corresponding generation sequence is

$$
\begin{gathered}
x^{(1)}=\left(x^{(1)}(1), x^{(1)}(2), \ldots, x^{(1)}(n)\right) \\
\left(x^{(1)}(k)=\sum_{i=1}^{k} x^{(0)}(i), k=1,2, \ldots, n\right) .
\end{gathered}
$$

GM $(1,1)$ model uses differential method to build a single variable first-order differential equation where the a is the development coefficient and the $b$ is the grey action 
quantity, the original form is

$$
\mathrm{x}_{0}(\mathrm{k})+\mathrm{ax}_{1}(\mathrm{k})=\mathrm{b} .
$$

Close to the average generation sequence is

$$
\begin{gathered}
\mathrm{Z}_{1}=\left(\mathrm{z}_{1}(2), \mathrm{z}_{1}(3), \ldots, \mathrm{z}_{1}(\mathrm{n})\right) \\
\mathrm{z}_{1}(\mathrm{k})=\frac{1}{2}\left(\mathrm{x}_{1}(\mathrm{k})+\mathrm{x}_{1}(\mathrm{k}-1)\right), \mathrm{k}=1,2, \ldots, \mathrm{n} .
\end{gathered}
$$

The basic form of GM $(1,1)$ model is

$$
\mathrm{x}_{0}(\mathrm{k})+\mathrm{az}_{1}(\mathrm{k})=\mathrm{b} .
$$

Whitened equation which is whitened by first-order grey differential equation is

$$
\frac{\mathrm{dx}_{1}}{\mathrm{dt}}+\mathrm{ax}_{1}=\mathrm{b}
$$

The solution is

$$
x_{1}(t)=\left(x_{1}(1)-\frac{b}{a}\right) e^{-a t}+\frac{b}{a} .
$$

\section{The Proposed Method AND Design}

The technical route of this paper is shown below in Figure 1 .

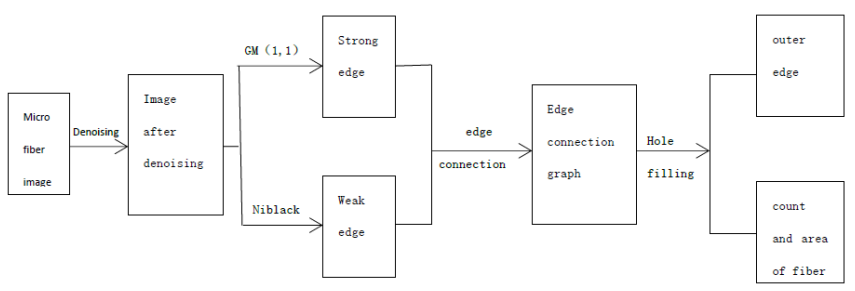

Figure 1. The technical route is composed of image denoising, strong edge extraction, weak edge extraction, strong and weak edge connection, hole filling.

\section{A. Strong edge extraction}

The grey prediction model is based on the known data and unknown parameters in the system to establish the concrete model of the system development law [7]. According to the fiber grey value and the characteristics that grey value difference between the background region and the interior of the fiber is relatively large; the grey prediction model is used to extract the strong edge of the fiber edge. The model algorithm is based on grey value difference between the predicted image and the actual grey value to judge whether the point is the edge point.

Set the grey value of point $I(i, j)$ is $g(i, j)$ on the $M \times$ $\mathrm{N}$ size of image. Based on the selection scheme of sequence points of grey prediction model and the grey feature of fiber images, the grey value $g(i, j)$ of each pixel $I(i, j)(i=$ $1,2,3, \ldots \mathrm{M}, \mathrm{j}=1,2,3 \ldots, \mathrm{N})$ and its neighboring pixels in the image are regarded as the discrete initial sequence taken by the continuous variable in the process of the change of the grey prediction model. Selecting the 12 points mask sequence as shown in Figure 2 to predict the grey value $g(i, j)$ of pixelsI $(i, j)$. The original sequence of the image is processed into a regular sequence which is suitable for the grey modeling by the data accumulation, so that the grey value of the center point is calculated by using the $\operatorname{GM}(1,1)$ differential equation and the prediction image II is formed. After getting the predicted value series, having data reduction to obtain the actual predicted data of the point, and obtaining the error image III which is equaled to the minus between the original image I and the prediction imageII. The grey value of each point in the image is $\zeta(i, j)$. Setting the threshold is $\mathrm{T}$ according to the grey level histogram of the error image. If $\xi(\mathrm{i}, \mathrm{j})>\mathrm{T}$, setting the grey value $B(i, j)=1$ of the point in strong edge of the binary image, otherwise $B(i, j)=0$. Thus the binary image of the strong edge can be obtained by the grey prediction model.

\begin{tabular}{|l|c|c|c|c|}
\hline & & $g(i-2, j)$ & & \\
\hline & $g(i-1, j-1)$ & $g(i-1, j)$ & $g(i-1, j+1)$ & \\
\hline$g(i, j-2)$ & $g(i, j-1)$ & $g(i, j)$ & $g(i, j+1)$ & $g(i, j+2)$ \\
\hline & $g(i+1, j-1)$ & $g(i+1, j)$ & $g(i+1, j+1)$ & \\
\hline & & $g(i+2, j)$ & & \\
\hline
\end{tabular}

Figure 2. In order to ensure the edge information can be detected in an arbitrary direction, the image is expanded to be $(\mathrm{M}+4)^{*}(\mathrm{~N}+4)$.

\section{B. Weak Edge Extraction}

After the pretreatment of Gauss filter, the interference of the background grey level gradient value is still existed in micro fiber image. The point selection scheme of the grey prediction model is improved to make the detection of the strong edge having more abundant information and according to the strong edge can also accurately identify fiber edge position, but strong edge exist certain fracture phenomenon which cannot satisfy the requirement of complete fiber edge [8]. To extract complete fiber edge information and overcome the incomplete edge of the edge extraction based on the grey prediction model, the Niblack algorithm is used to extract the weak edge of the fiber. The algorithm is a local dynamic threshold algorithm. Determine the center coordinates $(x, y)$ and the $r \times r$ range around the neighborhood. Make $g(x, y)$ as the grey value of the center coordinates. The result after binarization isb $(x, y)$, and calculating the average grey level $\mathrm{m}(\mathrm{x}, \mathrm{y})$ and the standard varianced $(x, y)$. The threshold value of the center point is calculated according to the mean and the variance, where $\alpha$ is the correction factor.

$$
t(x, y)=\alpha \times d(x, y)+m(x, y) .
$$

Then having the binarization of the center point by the threshold which has already been calculated to adaptively determine the threshold value in the different areas of the image and extract more detailed information of the image edge.

$$
b(x, y)=\left\{\begin{array}{lr}
0, & g(x, y) \leq t(x, y) \\
255, & g(x, y)>t(x, y)
\end{array} .\right.
$$

\section{Strong and weak edge connection}


After the strong threshold value is determined, there is certain edge incomplete phenomenon of the strong edge detection based on the grey prediction model of micro fiber. The weak edge of micro fibers which is extracted by Niblack algorithm has more detailed edge information. Combined with the strong edge and the weak edge of the micro fiber image, if the position of a pixel $I(i, j)$ belongs to the strong edge, then the pixel is reserved for the edge pixel; if the position of a pixel I $(\mathrm{i}, \mathrm{j})$ does not belong to the weak edge, then the pixel is discarded; if the position of a pixel $I(i, j)$ belongs to the weak edge but does not belong to the strong edge, then the pixel $I(i, j)$ is reserved for the edge pixel only when the 8 neighborhood points around itself within connection with the point which is on the strong edge. The recursive thinking is integrated into the strong and weak edge connection at the same time. The pixel of the weak edge which connects with the pixel of the strong edge joins the strong edge and repeat before operation. The algorithm which is collecting the pixel of the weak edge in the pixel of the strong edge constantly achieves the strong and weak edge connection. Removing false edge and preserving real edge in non-maximum inhibition results makes the connecting edge not only complete accurate and real consistent, but also greatly enhanced immunity.

\section{EXPERIMENT AND ANALYSIS}

\section{A. Edge Detection Effect}

To verify the performance of algorithm, selecting a large number of single fibers and multi-fiber micro fibers as the experimental sample, wherein the single fibers are divided into 1 font, Criss-cross, triangle, five-pointed star, Hollow shape, VY-shaped and W-shaped seven types after efficient algorithm design.

TABLE 1. EFFECT OF STRONG EDGE DETECTION ON FIVE ANGLE TYPE SINGLE FIBER WITH THRESHOLD

\begin{tabular}{|c|c|c|c|c|c|c|c|c|}
\hline $\begin{array}{l}\text { original } \\
\text { image }\end{array}$ & fiber & $\begin{array}{l}\text { average } \\
\text { residual }\end{array}$ & accuracy & $\begin{array}{l}1 \text { *average } \\
\text { residual }\end{array}$ & $\begin{array}{l}2 * \text { average } \\
\text { residual }\end{array}$ & $\begin{array}{l}\text { *average } \\
\text { residual }\end{array}$ & $\begin{array}{l}\text { 4*average } \\
\text { residual }\end{array}$ & $\begin{array}{l}\text { \%*average } \\
\text { residual } \\
\end{array}$ \\
\hline & & $4.9866 \%$ & $95.0133 \%$ & & & & & \\
\hline & & $6.6715 \%$ & $93.3284 \%$ & & & & & \\
\hline & & $5.2818 \%$ & $94.7181 \%$ & & & & & \\
\hline & & $5.8939 \%$ & $94.1060 \%$ & & & & & \\
\hline & & $8.0127 \%$ & $91.9872 \%$ & & & & & \\
\hline & & $5.0453 \%$ & $94.9546 \%$ & & & & & \\
\hline & & & & & . & & & \\
\hline
\end{tabular}

In the process of detection for the strong edge, the fivepointed star type of single fiber image as an example. Strong edge detection effect is shown in Table 1 in the case of different thresholds.

The traditional edge detection algorithm is used to obtain the false, discontinuous edges and cannot effectively extract the edge of the adhesive fibers. In this paper, the edge detection algorithm based on the gray system theory can be used to extract the accurate and continuous micro fiber edge. The edge detection effect diagram is shown in Figure 3 .

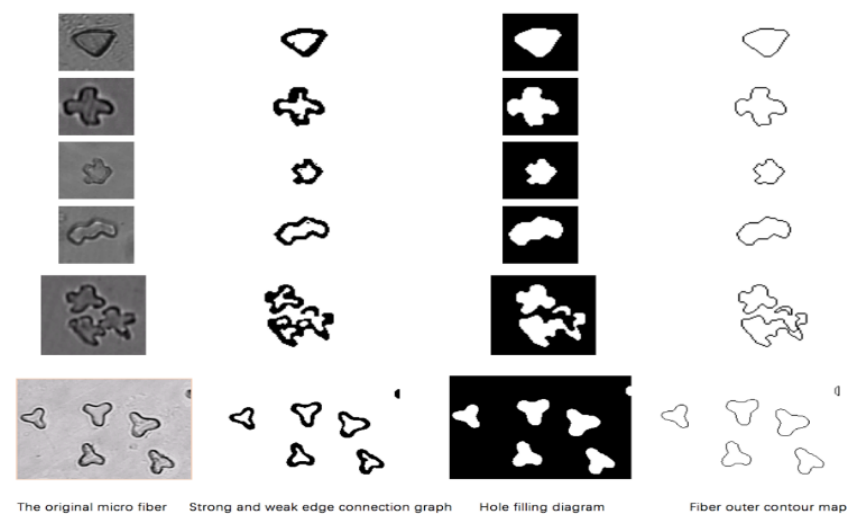

Figure 3. In the process of the strong and weak edge connection, the complete edge of the fiber can be obtained by setting the number of recursive iterationN $=2$. 


\section{B. The Count and Area of Target Fiber}

After getting an accurate and complete contour of the micro fiber images by the strong and weak edge connection, traversing all the contours of the target fiber and getting the area of current target contour. The result is shown in Figure 4.
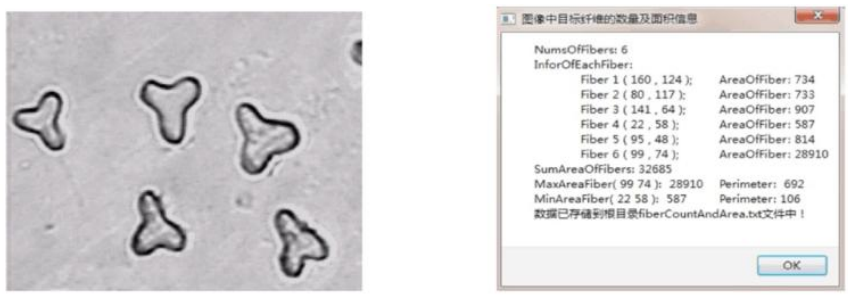

Figure 4. Left Image is the target fiber. Right image is the count and area effect chart.

\section{System Design}

Based on the graphical user interface application framework Qt, a fiber edge detection system is developed. The system which depends on micro fiber edge detection algorithm based on grey system theory contains the image related transformation, image enhancement process, edge detection and contour tracing, image information extraction, image segmentation and counting, grey system theory application of fiber image six core module.

\section{CONCLUSION}

The edge detection problem of micro fiber image is attributed to the uncertainty and poor information system. With the combination of the traditional edge detection algorithm and the grey system theory, a new edge detection algorithm is proposed, and the key technologies of the algorithm design and system design are described. The mathematical model of grey system theory is discussed, the strong edge and weak edge extraction of micro fiber image are analyzed, and the flow of algorithm of the strong and weak edge connection is described. The experimental results show that the effectiveness of the algorithm which can provide a good basis for the separation of fibers.

\section{REFERENCES}

[1] Haiping Huang, Shichao Huang, Jiutian Chen, Ruchuan Wang, Jianhui Xiong. An image information hiding algorithm based on grey system theory [J]. Int. J. Commun. Syst. (2014)

[2] LianFu Han, Wen Yan Tang, YongMeng Liu, Jun Wang, ChangFeng $\mathrm{Fu}$. Evaluation of measurement uncertainty based on grey system theory for small samples from an unknown distribution [J]. Science China Technological Sciences (2013)

[3] Sifeng Liu, Jeffrey Forrest, Yingjie Yang. A brief introduction to grey systems theory [J]. Grey Systems: Theory and Application (2012)

[4] Zhigang Zhou, Nong Sang. Image Edge Detection Algorithm Based on Grey System Theory [J]. International Journal of Digital Content Technology and its Applications (2012)

[5] A. Vasilevskiy, K. Siddiqi. Flux Maximizing Geometric Flows. IEEE Trans. Pattern Analysisi Machine Intelligence (2002)

[6] Siddiqi K, Bouix S, Tannenbaum A, et al. Hamilton-Jacobi Skeletons [J]. International Journal of Computer Vision (2002)
[7] Dimitrov P, Phillips C, Siddiqi K. Robust and efficient skeletal graphs [M]// IEEE (2000)

[8] Mayya N, Rajan V T. Voronoi diagrams of polygons: a framework for shape representation [J]. Journal of Mathematical Imaging \& Vision (1996) 